Recepción: 20 / 04 / 2017

Aceptación: 20 / 05 / 2017

Publicación: 15 / 06 / 2017

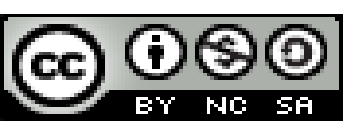

Ciencias de la Educación

Artículo de Investigación

\title{
Programación orientada a objetos, aprendizaje basado en lego mindstorms y propuesta de guía para la operacionalización
}

\section{Object-oriented programming, lego mindstorms based learning and proposal for a guide to the operationalization}

\section{Orientada a objeto, com base em lego mindstorms aprendizagem e proposta para o guia de programação operacionalização}

Juan C. Ramos-Romero ${ }^{\mathrm{I}}$ juanka.ramos@gmail.com

Janet E. Pazmiño-Ramírez ${ }^{\text {II }}$ janetpazmino@hotmail.com

Silvia A. Medina-Anchundia ${ }^{\text {III }}$ silvia.medinaa@ug.edu.ec

Correspondencia: janetpazmino@ hotmail.com

Ingeniero en Sistemas Computacionales; Magister en Docencia y Gerencia en Educación Superior; Universidad de Guayaquil, Guayaquil, Ecuador.

II. Magister en Diseño Curricular; Profesora Especialización Educación Primaria, Profesora de Segunda Enseñanza en la Especialización de Comercio y Administración, Licenciada en Ciencias de la Educación Especialización Educación Primaria, Universidad de Guayaquil, Guayaquil, Ecuador.

III. Magister en Administración de Empresas; Ingeniera Comercial; Universidad de Guayaquil, Ecuador, Guayaquil, Ecuador. 


\section{Resumen}

La tecnología se ha convertido de una herramienta productiva a algo que forma parte de la vida diaria, es una necesidad estar conectados y utilizar dispositivos electrónicos móviles o estacionales, sobre los cuales se ejecutan aplicaciones, éstas deben ser desarrolladas usando lenguajes y metodologías de programación. La programación orientada a objetos es la tendencia actual y su aprendizaje tiene una curva muy pronunciada dado los conceptos abstractos que se maneja en su entorno, al intentar emular los objetos o cosas de la vida real en unidades lógicas de programación llamadas clases, luego se crean instancias y producen objetos únicos. La propuesta de la tesis presenta una herramienta didáctica que proviene de los principios de la robótica conocida como Lego Mindstorms para el aprendizaje y comprensión de la Programación Orientada a Objetos. En este trabajo se plantean hipótesis y su modelo lógico se probará a través de instrumento de investigación del tipo descriptiva y explicativa. La investigación va a ser Cuali-Cuantitativa, puesto que se va a encuestar a los estudiantes de tercer ciclo de la carrera Ingeniería en Sistemas Computacionales de la Universidad de Guayaquil. El producto final es una guía para el uso y operacionalización de Lego Mindstorms que facilitará al aprendizaje de Programación Orientada a Objetos con lo cual se podrá desarrollar aplicaciones empresariales, ofimáticas, colaborativas y móviles que aportarán con el crecimiento del mercado de software ecuatoriano y de la comunidad tecnológica.

Palabras claves: Orientada a objetos; aprendizaje; Lego Mindstorm; operacionalización. 


\begin{abstract}
The technology has become a productive tool to something that is part of daily life, is a must be connected and mobile or seasonal use electronic devices on which applications are running, they must be developed using programming languages and methodologies. Object-oriented programming is the current trend and the learning curve has been a steep abstract concepts that is handled in your environment, to try to emulate objects or things in real life programming into logical units called classes, then create instances and produce unique objects. The proposal of the thesis presents a didactic tool that comes with the principles of robotics known as Lego Mindstorms for learning and understanding of Object Oriented Programming. In this work hypothesis and its logical model is tested through research tool descriptive and explanatory type. The research will be qualitative and quantitative, as to be surveying graduate students career in Computer Systems Engineering from the University of Guayaquil. The final product is a guide to the use and operationalization of Lego Mindstorms will facilitate the learning of Object- Oriented Programming with which it can develop business, office, and mobile collaborative applications that contribute to the growth of the software market and the Ecuadorian technology community.
\end{abstract}

Key words: Object oriented programming; learning; Lego Mindstorms; operationalisation 


\section{Resumo}

A A tecnologia tornou-se uma ferramenta produtiva para algo que faz parte da vida cotidiana, é um deve ser conectado e usar dispositivos electrónicos móveis ou sazonais nos quais aplicativos são executados, devem ser desenvolvidos usando linguagens e metodologias de programação. Programação orientada a objetos é a tendência atual e aprendizagem tem uma curva acentuada como conceitos abstratos manipulados em seu ambiente, tentando imitar os objetos ou coisas da vida real em unidades de programação lógicas chamadas classes, em seguida, criar instâncias e produzir objetos únicos. A proposta da tese apresenta uma ferramenta de ensino que vem dos princípios da robótica Lego Mindstorms conhecidos como aprendizado e compreensão da OOP. Neste trabalho hipóteses são testadas e o modelo lógico é testado através de ferramenta de pesquisa descritiva e explicativa. A investigação será quali-quantitativa, uma vez que irá examinar a estudantes de graduação Carreira Computer Systems Engenharia da Universidade de Guayaquil. O produto final é um guia para o uso e operacionalização de Lego Mindstorms facilitará a OOP aprendizagem, assim, podem desenvolver negócios, automação de escritório, colaborativa e aplicações móveis que contribuem para o crescimento de software equatoriana mercado e comunidade tecnológica.

Palavras-chave: orientado a objetos; aprendizagem; colocar Mindstorm; operacionalização. 
Juan C. Ramos-Romero; Janet E. Pazmiño-Ramírez; Silvia A. Medina-Anchundia

\section{Introducción.}

Se están viviendo cambios acelerados de épocas históricas de la humanidad, hace poco se hablaba de la era de la información, en la cual la globalización y el acceso libre a las base de datos mundiales fueron marcando la pauta del aprendizaje, todos tienen acceso a la misma información, pero no todos la consumían de manera apropiada, esta idea evolucionó y se convirtió en la "sociedad del conocimiento", en donde la premisa era el conocimiento es poder, hoy ya no es suficiente tener solamente la información sino que hay la necesidad de procesarla y convertirla en el tal llamado y ansiado conocimiento.

Luego de tener el conocimiento, se lo debe compartir y es aquí en donde las redes sociales se han apoderado de las vidas de las personas, tanto que se puede saber el itinerario de una persona con tan solo revisar su "perfil". Esta necesidad imperiosa de compartir la información, hechos, sucesos, vivencias, capturas gráficas, trajo consigo la "tendencia de la conectividad", la cual abre las puertas a la siguiente era, denominada "el internet de las cosas".

Todas las etapas o eras de la evolución de los seres humanos, han venido acompañada con importantes avances tecnológicos, empezando con el fuego, las armas, la rueda, la pólvora pasando por las máquinas industriales, radio, electricidad, hasta llegar a la actualidad en donde los dispositivos electrónico sean estos fijos o móviles permiten estar comunicados, acortar las largas distancias (aunque también alargar las distancias cortas, pero para tratar ese tema se necesitaría otra tesis completa).

Toda esta revolución que ya no solo es industrial, sino revolución de la interacción va acompañada de tecnología, que está compuesta por dos partes muy importantes: el hardware y 
software. Entiéndase por "hardware" todo lo que se puede tocar, es decir el equipo o aparato electrónico en sí.

Con todo esto al "software", que es la parte fundamental de ésta investigación, esa parte intangible y muchas veces pasada por alto, pero sin la cual, cualquier dispositivo electrónico no sería más que un elegante y costoso pisapapeles, sin menospreciar al hardware, pero la relación y dependencia que tiene un componente del otro es tal que si se junta con la conectividad formarían la "santa trinidad tecnológica" que no pueden separarse y funcionan perfectamente juntas.

Ahora, el software debe ser construido, desarrollado o programado, cualquiera sea el término usado implica que se debe "picar código" para poder crear estas aplicaciones que ahora son el centro de nuestras vidas.

La programación hasta llegar a la era moderna también ha sufrido cambios y ha evolucionado desde la metodología lineal hasta convertirse en metodología orientada a objetos y en la actualidad se está trabajando en nuevas formas de desarrollo. 


\section{Materiales y métodos.}

\section{Población y muestra.}

Según Levin \& Rubin (1996), “Una población es un conjunto de todos los elementos que estamos estudiando, acerca de los cuales intentamos sacar conclusiones”. (pág. 67). Finalmente Cadenas (1974) dice. "Una población es un conjunto de elementos que presentan una característica común”. (pág. 38).

El tamaño de la población o universo es un factor fundamental en el proceso de investigación estadística y observando el origen educativo de la investigación la población viene dada por los estudiantes y docentes de la Carrera de Ingeniería en Sistemas de la Universidad de Guayaquil, para el caso específico del presente trabajo de investigación se va a considerar los estudiantes de tercer nivel que están tomando la materia Programación Orientada a Objetos y los docentes de la misma materia.

Cuando el número de elementos que conforma la población es extenso o muy grande, se puede considerar la población como infinita, en el caso de estudio presente se está frente a una población finita porque está formada por un número limitado e identificable de elementos, así como se lo describió en el párrafo anterior.

La Población para el presente trabajo de investigación, luego de obtener los datos, se lo ha definido de la siguiente forma.

La población investigada, viene dada por el número de estudiantes de Tercer Ciclo que toman la materia Programación Orientada a Objetos, así como sus respectivos docentes, es decir de 3 paralelos para las jornadas matutina, vespertina y nocturna. 
Dada que la población es finita y por el número de elementos se determina que es factible tomar al total de elementos presentados como parte de la población del presente trabajo de investigación.

A continuación, se contabiliza la población en el cuadro mostrado a continuación:

\begin{tabular}{|l|c|c|}
\hline \multicolumn{1}{|c|}{ Segmento } & Cantidad & Porcentaje \\
\hline Estudiantes & 120 & $96 \%$ \\
\hline Docentes & 5 & $4 \%$ \\
\hline TOTAL & 125 & $100 \%$ \\
\hline
\end{tabular}

\section{Cuadro \#1}

\section{Población}

Fuente: secretaría de la carrera de ingeniería en sistemas de la Universidad de Guayaquil

Por lo tanto, según se señaló en el cuadro $\mathrm{N}^{\circ} 5$ a 120 estudiantes y 5 docentes se le va a aplicar el instrumento de la encuesta diseñada para el presente trabajo de investigación.

\section{Métodos}

La importancia de la utilización de la metodología a emplearse en el presente trabajo de investigación, se fundamenta en la necesidad de realizar una sinergia de criterios que faciliten y optimicen la selección, organización y planteamientos de procedimiento y recursos factibles que permitan identificar las particularidades del problema y plantear las posibles soluciones. Entre los métodos y las técnicas a utilizarse cabe mencionar el método hipotético-deductivo, el método inductivo-deductivo y el método lógico. 
Juan C. Ramos-Romero; Janet E. Pazmiño-Ramírez; Silvia A. Medina-Anchundia

Instrumentos de investigación.

En el trabajo de investigación que se está presentando se utilizan los cuestionarios como instrumentos de la técnica de la encuesta

\section{Resultados.}

Análisis e interpretación de resultados de la encuesta a los docentes

Todo trabajo de investigación nace con la finalidad de estudiar y proponer una solución a un fenómeno al que se denomina problema, además se deben plantear objetivos y sobretodo una hipótesis, la misma que debe ser demostrada para ser comprobada o rechazada estadísticamente.

En relación a esta ardua tarea, se diseñó un cuestionario que, luego de ser validado por especialistas, se lo aplicó a una población determinada de informantes, lo cual se detalló en el capítulo anterior.

Luego de la recolección de la información mediante la encuesta se procedió a tabularla y procesarla mediante el software de cálculo y elaborar tablas estadísticas, para la compresión y análisis de los porcentajes obtenidos. La elaboración de gráficos para cada pregunta del cuestionario es un requisito fundamental y eje transversal del presente capítulo. Los resultados son presentados por cada ítem, según el orden que se encuentra en el instrumento.

A continuación, se presenta el resultado de la aplicación del cuestionario a los informantes: 
Resultados e interpretación de resultados de la encuesta a docentes.

\begin{tabular}{lcr}
\multicolumn{1}{c}{ ITEMS } & FRECUENCIA & PORCENTAJE \\
\hline Totalmente de Acuerdo & 0 & $0 \%$ \\
\hline De Acuerdo & 2 & $40 \%$ \\
No Opina & 0 & $0 \%$ \\
En desacuerdo & 3 & $60 \%$ \\
Total Desacuerdo & 0 & $0 \%$ \\
\multicolumn{1}{r}{ TOTAL } & $\mathbf{5}$ & $\mathbf{1 0 0 \%}$
\end{tabular}

\section{Cuadro \#2}

Item\# 1. Conceptos de programación orientada a objetos son complejos de comprender.

El $60 \%$ de los docentes encuestados no están de acuerdo que los conceptos de Programación orientada a objetos son complejos, mientras que el $40 \%$ opina lo contrario.

Los conceptos de POO son abstractos, puesto que su objetivo es emular las cosas de la vida cotidiana y convertirlos en elementos de programación, y se basa en propiedades y características no tangibles.

Se nota que la mayoría de los docentes piensan que los conceptos de orientación a objetos pueden ser comprendidos por los estudiantes sin mayor dificultad, aunque una cantidad no despreciable no piensa igual, lo que se debe considerar en el análisis.

\begin{tabular}{lcr}
\multicolumn{1}{r}{ ITEMS } & FRECUENCIA & PORCENTAJE \\
\hline C ++ & 0 & $0 \%$ \\
Java & 4 & $80 \%$ \\
SmallTalk & 0 & $0 \%$ \\
C\# & 1 & $20 \%$ \\
VB.Net & 0 & $0 \%$ \\
\multicolumn{1}{r}{ TOTAL } & $\mathbf{5}$ & $\mathbf{1 0 0 \%}$
\end{tabular}

\section{Cuadro \#3}

Item\# 2. Lenguaje orientado a objetos más relevante Fuente: Resultado de la Investigación. 
Los docentes encuestados, en el $80 \%$, se pronunciaron indicando que el lenguaje de programación orientado a objetos más relevante es java, mientras que en segundo lugar en importancia es C\#, del resto de lenguajes no se pronunciaron.

Los lenguajes de programación han evolucionado en los últimos años. Java es el lenguaje que lleva la bandera, existen más de 3 billones de equipos electrónicos actualmente ejecutando java y se encuentra en un proceso de mejora continua.

En la actualidad todos los dispositivos necesitan ejecutar aplicaciones y éstas deben estar desarrolladas con algún lenguaje de programación, java es el favorito de la industria por su versatilidad, independencia de arquitectura, multiplataforma y alto rendimiento, además es un lenguaje $100 \%$ orientado a objetos que cumple con todas las características y es motivo de estudio en la materia Programación Orientada a Objetos en la Carrera de Ingeniería en Sistemas.

\begin{tabular}{lcr}
\multicolumn{1}{c}{ ITEMS } & FRECUENCIA & PORCENTAJE \\
\hline DEFINITIVAMENTE SI & 1 & $20 \%$ \\
SI & 3 & $60 \%$ \\
NO OPINA & 1 & $20 \%$ \\
NO & 0 & $0 \%$ \\
DEFINITIVAMENTE & & $0 \%$ \\
NO $\quad$ TOTAL & 0 & $\mathbf{1 0 0 \%}$
\end{tabular}

Fuente: Resultado de la Investigación. Cuadro \#4

\section{Item\# 3. Documentación adecuada de la fundamentación de la orientación a objetos}

Los resultados de las encuestas presentan que un total de $80 \%$ de los docentes indican que definitivamente se encuentra debidamente documentada la fundamentación teórica de la programación orientada a objetos. Algo poco usual se presentó con un $20 \%$ que prefirió no opinar del tema. 
La Programación orientada a Objetos necesita de una base sólida de conocimientos teóricos para que pueda ser aplicada de manera adecuada y de ésta manera aprovechar todas las ventajas que brinda.

Con esto los docentes indican que poseen guías, libros, manuales y documentos que son usados en sus clases y los estudiantes tienen acceso a los mismos. Además que la documentación es pertinente y adecuada.

\begin{tabular}{lcr}
\hline \multicolumn{1}{|c}{ ITEMS } & FRECUENCIA & PORCENTAJE \\
\hline Totalmente de Acuerdo & 3 & $60 \%$ \\
De Acuerdo & 1 & $20 \%$ \\
No Opina & 1 & $20 \%$ \\
En desacuerdo & 0 & $0 \%$ \\
Total Desacuerdo & 0 & $0 \%$ \\
\hline \multicolumn{2}{c}{ TOTAL } & $\mathbf{5}$ \\
Fuente: Resultado de la Investigación. \\
Cuadro \#5 \\
Item\# 4. Ayuda de la robótica al hombre en la educación superior
\end{tabular}

La gran mayoría de docentes de Programación Orientada a Objetos están de acuerdo con que la robótica ha contribuido al hombre en algunas áreas incluyendo la Educación Superior, porcentualmente el $80 \%$ coincide con la pregunta. Se repita que en un porcentaje mucho menor prefiere no emitir su criterio acerca de lo consultado.

El hombre siempre se encuentra en la búsqueda de nuevas fronteras, lo cual se ha derivado en la creación de herramientas, como robots, que lo ayuden con las tareas complejas o peligrosas, por ejemplo el ensamblaje, manipulación de sustancias tóxicas, excavación, desarme de bombas, etc.

Pero el resultado va más allá de eso, puesto que se puede inferir que están de acuerdo que la robótica puede ayudar en el proceso de enseñanza-aprendizaje orientado a la Educación Superior y abre la puerta a la aplicación de nuevas herramientas didácticas como el uso de robótica. 


\begin{tabular}{lcr}
\multicolumn{1}{c}{ ITEMS } & FRECUENCIA & PORCENTAJE \\
\hline Totalmente de Acuerdo & 1 & $20 \%$ \\
De Acuerdo & 4 & $80 \%$ \\
No Opina & 0 & $0 \%$ \\
En desacuerdo & 0 & $0 \%$ \\
Total Desacuerdo & 0 & $0 \%$ \\
\multicolumn{1}{c}{ TOTAL } & $\mathbf{5}$ & $\mathbf{1 0 0 \%}$
\end{tabular}

\section{Fuente: Resultado de la Investigación.}

Cuadro \#6

\section{Item\# 5. Falta de herramientas didácticas y robóticas en el aprendizaje de programación}

El resultado de ésta pregunta es muy particular puesto que el $20 \%$ está completamente de acuerdo y el $80 \%$ se encuentra de acuerdo, sumando en total el $100 \%$ de los encuestados que opinan que hace falta herramientas didácticas y robóticas en el proceso de enseñanza-aprendizaje de programación orientada a objetos.

Tomando en cuenta que la población está conformada por docente de la materia Programación Orientada Objetos se puede notar que no se encuentran satisfechos con las herramientas actuales y están buscando otros tipos de instrumentos con los cuales puedan llegar al estudiante.

La Didáctica, laboratorios y práctica deben ir de la mano para garantizar el aprendizaje de programación de los estudiantes.

\begin{tabular}{lrr}
\multicolumn{1}{c}{ ITEMS } & FRECUENCIA & PORCENTAJE \\
\hline Totalmente de Acuerdo & 5 & $100 \%$ \\
De Acuerdo & 0 & $0 \%$ \\
No Opina & 0 & $0 \%$ \\
En desacuerdo & 0 & $0 \%$ \\
Total Desacuerdo & 0 & $0 \%$ \\
\multicolumn{1}{r}{$\quad$ TOTAL } & $\mathbf{5}$ & $\mathbf{1 0 0 \%}$
\end{tabular}

Cuadro \#7

Item\# 6. Armar y programar un robot de lego influye positivamente en el aprendizaje 
En éste ítem se puede observar claramente el apoyo del $100 \%$ de los docentes de programación orientada a objetos en que armar y enviar acciones a un robot mediante comandos influiría positivamente en el aprendizaje.

Las formas de aprendizaje van cambiando constantemente y el uso de robótica complementaría la explicación teórica de los conceptos e incentivaría el descubrimiento e investigación de los estudiantes.

Queda muy clara la opinión de los docentes, en donde evidencia el apoyo al uso de herramientas robóticas con las cuales se evidencia de forma práctica comandos, diseño de clases, objetos, líneas de programación o codificación que puede aportar positivamente a la clase y al desenvolvimiento de los estudiantes.

\begin{tabular}{|c|c|c|}
\hline ITEMS & FRECUENCIA & PORCENTAJE \\
\hline Totalmente de Acuerdo & 2 & $40 \%$ \\
\hline De Acuerdo & 3 & $60 \%$ \\
\hline No Opina & 0 & $0 \%$ \\
\hline En desacuerdo & 0 & $0 \%$ \\
\hline Total Desacuerdo & 0 & $0 \%$ \\
\hline TOTAL & 5 & $100 \%$ \\
\hline
\end{tabular}

El $100 \%$ de los informantes se encuentran de acuerdo en que personas que usan legos desempeñan funciones técnicas y científicas.

Estudios recientes de una universidad muy reconocida publicó los resultados de una investigación en donde concluye que las personas, o en ese caso los niños, que utilizan juguetes de armar y construir (por ejemplo los Legos) son más propensos, en el futuro, de realizar actividades 
técnicas o de ingeniería que los que juegan con otro tipo de artefactos como muñecos, carros y demás.

Este criterio mayoritario por parte de los catedráticos reafirma que los implementos tipo Lego contribuyen positivamente en el aprendizaje y puede ser una herramienta didáctica de mucha utilidad para los procesos cognoscitivos.

\begin{tabular}{lcr}
\multicolumn{1}{r}{ ITEMS } & FRECUENCIA & PORCENTAJE \\
\hline Definitivamente $\mathrm{Si}$ & 4 & $80 \%$ \\
$\mathrm{Si}$ & 1 & $20 \%$ \\
No Opina & 0 & $0 \%$ \\
No & 0 & $0 \%$ \\
Definitivamente No & 0 & $0 \%$ \\
\multicolumn{1}{r}{ TOTAL } & $\mathbf{5}$ & $\mathbf{1 0 0 \%}$
\end{tabular}

\section{Cuadro \#9}

\section{Item\# 8. Información sobre lego Mindstorms y su aplicación en la educación}

Esta pregunta en donde se consulta, si desean obtener más información acerca de Lego Mindstorms y su aplicación en las ciencias de la educación se cuenta con una mayoría absoluta con el $100 \%$ a favor en donde el $80 \%$ indica que definitivamente si desearía y el $20 \%$ restante también responde de manera afirmativa.

Como se ha podido evidenciar el uso de nuevas herramientas didácticas y tecnológicas que apoyen el proceso de enseñanza-aprendizaje es bien recibido por parte de los docentes, puesto que aportan positivamente en el desenvolvimiento del estudiante en el aula de clases e incentiva la investigación y descubrimiento.

Es importante recalcar que Lego Mindstorms combina robótica con programación de acciones y esto es el factor fundamental por la cual los docentes se encuentran deseosos de conocer más aun sobre esta novedad que puede convertirse en aliado en la educación. 


\begin{tabular}{|c|c|c|}
\hline ITEMS & FRECUENCIA & PORCENTAJE \\
\hline Definitivamente $\mathrm{Si}$ & 0 & $0 \%$ \\
\hline $\mathrm{Si}$ & 2 & $40 \%$ \\
\hline No Opina & 1 & $20 \%$ \\
\hline No & 2 & $40 \%$ \\
\hline Definitivamente No & 0 & $0 \%$ \\
\hline TOTAL & 5 & $100 \%$ \\
\hline
\end{tabular}

La opinión de los informantes fue equilibrada en este ítem, en cuanto el $40 \%$ si conocía sobre los lenguajes de programación que se usa en Lego Mindstorms, mientras que el mismo porcentaje, es decir el 40\%, no conocía. Un 20\% de los informantes prefirieron No opinar por lo que se tiene un $80 \%$ el cual aportó sus consideraciones a la encuesta.

Lego Mindstorms soporta diversos lenguajes de programación como C, C\#, java, entre otros lo cual es importantísimo, ya que el hecho de no estar atado a una sola plataforma crea diversidad de conocimiento y opciones al momento de diseñar, con esto no es relevante el lenguaje a usar sino como se puede aplicar la herramienta al proceso de enseñanza-aprendizaje de la materia Orientada a Objetos.

\begin{tabular}{lcr}
\multicolumn{1}{c}{ ITEMS } & FRECUENCIA & PORCENTAJE \\
\hline Totalmente de Acuerdo & 2 & $40 \%$ \\
De Acuerdo & 3 & $60 \%$ \\
No Opina & 0 & $0 \%$ \\
En desacuerdo & 0 & $0 \%$ \\
Total Desacuerdo & 0 & $0 \%$ \\
\multicolumn{1}{r}{ TOTAL } & $\mathbf{5}$ & $\mathbf{1 0 0 \%}$
\end{tabular}

Cuadro \#11

Item\# 10. Facilidad de aprendizaje de programación con el uso de lego Mindstorms y su guía 
Al momento de consultar con los docentes si piensan que el aprendizaje se facilita con el uso de Lego Mindstorms con su respectiva guía se tuvo el $100 \%$ de apoyo, dividido en $40 \%$ que se encontraban Totalmente de Acuerdo y el resto está de Acuerdo.

Se puede confirmar el apoyo a la utilización de Lego Mindstorms como herramienta robótica didáctica para el aprendizaje de Programación Orientada a Objetos en la Universidad de Guayaquil.

Con estos resultados se puede confirmar la hipótesis planteada para el uso de la herramienta mencionada en el aula de clases como apoyo para el proceso enseñanza-aprendizaje, por lo tanto es altamente recomendado por los docentes que respondieron a la encuesta.

\begin{tabular}{|lcr|}
\multicolumn{1}{c}{ ITEMS } & FRECUENCIA & PORCENTAJE \\
\hline Totalmente de Acuerdo & 3 & $60 \%$ \\
De Acuerdo & 2 & $40 \%$ \\
No Opina & 0 & $0 \%$ \\
En desacuerdo & 0 & $0 \%$ \\
Total Desacuerdo & 0 & $0 \%$ \\
\multicolumn{1}{r}{ TOTAL } & $\mathbf{5}$ & $\mathbf{1 0 0 \%}$
\end{tabular}

\section{Cuadro \#12}

Item\# 11. Apoyo al uso de lego Mindstorms en el aprendizaje de programación orientada a objetos

En la última pregunta de la encuesta, la más importante para la investigación, se puede evidenciar que el $100 \%$ de los docentes apoyan el uso de Lego Mindstorms en el aprendizaje de Programación Orientada a Objetos.

El $40 \%$ de los encuestados estuvieron de acuerdo y el $60 \%$ de los docentes mencionaron estar Totalmente de Acuerdo con la aplicación de Mindstorms. Ningún informante estuvo en desacuerdo, lo que muestra claramente el criterio de los catedráticos. 
El uso de este tipo de herramientas robóticas en el proceso metodológico de clase es altamente recomendable en el proceso de enseñanza-aprendizaje, así lo piensan los docentes encuestados, por lo tanto, aprueban la hipótesis planteada dentro del trabajo de investigación.

Análisis e interpretación de resultados de la encuesta a los estudiantes.

\begin{tabular}{lcr}
\multicolumn{1}{c}{ ITEMS } & FRECUENCIA & PORCENTAJE \\
\hline Totalmente de Acuerdo & 11 & $9,17 \%$ \\
De Acuerdo & 49 & $40,83 \%$ \\
No Opina & 22 & $18,33 \%$ \\
En desacuerdo & 28 & $23,33 \%$ \\
Total Desacuerdo & 10 & $8,34 \%$ \\
\multicolumn{1}{r}{ TOTAL } & $\mathbf{1 2 0}$ & $\mathbf{1 0 0 \%}$
\end{tabular}

\section{Cuadro \#13}

Item\# 1. Conceptos de programación orientada a objetos son complejos de comprender

El 50\% de los estudiantes encuestados apoyan que los conceptos de Programación Orientada a Objetos son abstractos y por lo tanto complejos de entender, mientras que el $31,66 \%$ no comparte esa afirmación.

Por otro lado, un 18,33\% prefiere no opinar sobre el tema.

Se puede concluir que la mitad de los estudiantes están de acuerdo con la consulta realizada, aunque no es la mayoría es un porcentaje considerable, por lo que se debe tener en cuenta para el presente trabajo de investigación. 


\begin{tabular}{lcr}
\multicolumn{1}{r}{ ITEMS } & FRECUENCIA & PORCENTAJE \\
\hline Definitivamente $\mathrm{Si}$ & 11 & $9,17 \%$ \\
$\mathrm{Si}$ & 58 & $48,33 \%$ \\
No Opina & 22 & $18,33 \%$ \\
No & 29 & $24,17 \%$ \\
Definitivamente No & 0 & $0,00 \%$ \\
\multicolumn{1}{r}{ TOTAL } & $\mathbf{1 2 0}$ & $\mathbf{1 0 0 \%}$
\end{tabular}

\section{Cuadro \#14}

\section{Item\# 2. Conocimiento de los pilares fundamentales de la programación orientada a objetos}

Más del 55\% de los informantes indican que conocen los pilares fundamentales de la Programación Orientada a Objetos. El 24,17\% indica que no los conoce y el 18,33\% No opina nada al respecto.

Características como la Herencia, Polimorfismo, Abstracción y Encapsulamiento son los pilares fundamentales de la Programación Orientada a Objetos, y son la base para poder conocer y obtener provecho de ésta metodología, que se basa en emular cosas del mundo real y llevarla al mundo programático.

El conocimiento de las bases teóricas es de suma importancia y se cuenta con un alto porcentaje de estudiantes encuestados que la conocen, esto ayuda como plataforma para poder continuar con el trabajo de investigación puesto que va a desarrollar sobre estudiantes con fundamentos.

\begin{tabular}{|c|c|c|}
\hline ITEMS & FRECUENCIA & PORCENTAJE \\
\hline Definitivamente $\mathrm{Si}$ & 8 & $6,67 \%$ \\
\hline $\mathrm{Si}$ & 56 & $46,67 \%$ \\
\hline No Opina & 35 & $29,17 \%$ \\
\hline No & 20 & $16,66 \%$ \\
\hline Definitivamente No & 1 & $0,83 \%$ \\
\hline TOTAL & 120 & $100 \%$ \\
\hline
\end{tabular}

\section{Cuadro \#15}

Item\# 3. Explicar características más importantes de la programación orientada a objetos 
De nuestra población de estudiantes el 46,67\% indica que Si puede explicar y el 6,67\% que definitivamente Si puede explicar las características más importantes de la Programación Orientada a Objetos, dando un total de, aproximadamente, 54\% que respondieron de manera afirmativa.

Por lo contrario el $17,49 \%$ de los informantes indican que no pueden explicar las características. Finalmente, un porcentaje considerable, el 29,17\% No emite opinión respecto al tema en cuestión.

La Programación Orientada a Objetos posee importantes características como la reutilización, recolector de basura, librerías, ocultación, herencia, composición, etc. Que se deben conocer para poder hacer uso de la misma de una manera adecuada para aprovechar todas las ventajas que brinda.

\begin{tabular}{lc|rr} 
& ITEMS & FRECUENCIA & PORCENTAJE \\
\hline C ++ & 20 & $16,67 \%$ \\
Java & 78 & $65,00 \%$ \\
SmallTalk & 2 & $1,66 \%$ \\
C\# & 11 & $9,17 \%$ \\
VB.Net & 9 & $7,50 \%$ \\
& & $\mathbf{1 2 0}$ & $\mathbf{1 0 0 \%}$ \\
\multicolumn{4}{c}{ Item\# 4. Lenguaje orientado a objetos más relevante }
\end{tabular}

A los estudiantes se les consultó sobre los lenguajes de programación orientados a objetos más importantes y el 65\% coinciden que es java, el lenguaje de SUN que luego pasó a ser propiedad de Oracle.

En segundo lugar se tiene el padre de java y de muchos otros lenguajes $\mathrm{C}++$ con un apoyo del 16,67\%, algo que parece lógico luego que han revisado éste lenguaje en los ciclos anteriores. En 
menor porcentaje se tiene a C\# y VB.Net, ambos lenguajes de Microsoft que forman parte del paquete .Net Framework, con el 9,17\% y 7,50\% respectivamente.

Como resultado de la aplicación del cuestionario Java es el lenguaje más importante, dato que concuerda con la opinión de los docentes y se convierte en un pilar fundamental del presente trabajo de investigación, esta información es de suma importancia y se convierte en un criterio para la elaboración y formulación de la propuesta.

\begin{tabular}{|c|c|c|}
\hline ITEMS & FRECUENCIA & PORCENTAJE \\
\hline Definitivamente $\mathrm{Si}$ & 14 & $11,67 \%$ \\
\hline $\mathrm{Si}$ & 36 & $30,00 \%$ \\
\hline No Opina & 45 & $37,50 \%$ \\
\hline No & 25 & $20,83 \%$ \\
\hline Definitivamente No & 0 & $0,00 \%$ \\
\hline TOTAL & 120 & $100 \%$ \\
\hline
\end{tabular}

Resultado de la encuesta el $41,67 \%$ de los informantes opinan que los fundamentos de programación orientada a objetos se encuentran claramente documentados en los libros, textos, artículos o internet grafía. En cambio el 20,83\% piensa lo contrario, es decir, opina que no está claramente documentada.

De la misma forma, en un alto porcentaje, que suman el 37,50\% los estudiantes encuestados decidieron no emitir su opinión.

La práctica se debe fundamentar en teoría, y para tal, necesita encontrarse debidamente documentada. Se debe recordar que se está en la era del conocimiento, en donde no basta simplemente tener acceso a la información, sino procesarla, asimilarla y convertirla en conocimiento propio. 


\begin{tabular}{lcr}
\multicolumn{1}{c}{ ITEMS } & FRECUENCIA & PORCENTAJE \\
\hline Totalmente de Acuerdo & 44 & $36,67 \%$ \\
De Acuerdo & 60 & $50,00 \%$ \\
No Opina & 13 & $10,83 \%$ \\
En desacuerdo & 3 & $2,50 \%$ \\
Total Desacuerdo & 0 & $0,00 \%$ \\
\multicolumn{1}{r}{ TOTAL } & $\mathbf{1 2 0}$ & $\mathbf{1 0 0 \%}$
\end{tabular}

\section{Cuadro \#18}

\section{Item\# 6. Determinar los fundamentos de POO para} facilitar los procesos de enseñanza-aprendizaje

Un contundente $86,67 \%$ piensa que deben determinarse los fundamentos de Programación Orientada a Objetos para facilitar el proceso de enseñanza-aprendizaje en la Carrera de Ingeniería en Sistemas y Networking. Esto se divide en 50\% de Acuerdo y el 36,67\% Totalmente de Acuerdo.

El 2,50\% de los informantes están en desacuerdo mientras que el 10,83\% No opina respecto al ítem consultado.

La enseñanza-aprendizaje es un proceso constante, cíclico y dinámico que va evolucionando continuamente alrededor del tiempo, por lo tanto debe ser apoyado y mejorado con documentación teórica que ayuden a su aplicación, esto se pretende obtener con el presente trabajo de investigación.

\begin{tabular}{lcr}
\multicolumn{1}{c}{ ITEMS } & FRECUENCIA & PORCENTAJE \\
\hline Totalmente de Acuerdo & 45 & $37,50 \%$ \\
De Acuerdo & 62 & $51,67 \%$ \\
No Opina & 8 & $6,67 \%$ \\
En desacuerdo & 4 & $3,33 \%$ \\
Total Desacuerdo & 1 & $0,83 \%$ \\
\multicolumn{1}{r}{ TOTAL } & $\mathbf{1 2 0}$ & $\mathbf{1 0 0 \%}$
\end{tabular}

Item\# 7. Falta de guías por materias influye en el aprendizaje y rendimiento 
Juan C. Ramos-Romero; Janet E. Pazmiño-Ramírez; Silvia A. Medina-Anchundia

La gran mayoría de los estudiantes que forman parte de la población están de acuerdo con que la falta de guías especializadas por materia influye en el aprendizaje y rendimiento, en total suman un $89,17 \%$

El 10\% faltante, aproximadamente, se reparte entre las persona que No Opinan, están en Desacuerdo y en Total Desacuerdo, en donde el No Opina obtiene el 6,67\% que no influye mayormente en el resultado del presente trabajo de investigación.

El libro guía es fundamental en el aprendizaje de cada materia, pero tener una guía por cada materia es el escenario ideal para el proceso enseñanza-aprendizaje, en ésta guía específica se coloca la parte teórica, ejemplos, ejercicios y talleres con el cual el estudiante tiene información precisa clase por clase.

\begin{tabular}{lcr}
\multicolumn{1}{c}{ ITEMS } & FRECUENCIA & PORCENTAJE \\
\hline Totalmente de Acuerdo & 8 & $6,67 \%$ \\
De Acuerdo & 42 & $35,00 \%$ \\
No Opina & 55 & $45,83 \%$ \\
En desacuerdo & 14 & $11,67 \%$ \\
Total Desacuerdo & 1 & $0,83 \%$ \\
\multicolumn{1}{r}{ TOTAL } & $\mathbf{1 2 0}$ & $\mathbf{1 0 0 \%}$
\end{tabular}

Cuadro \#20

Item\# 8.conocimiento acerca de los estilos de aprendizaje del ser humano

En ésta pregunta el porcentaje mayor lo obtuvieron los informantes que No opinaron, con un 45,83\%, lo cual indica que se desconoce del tema. Además, el 41,67\% se encuentran de acuerdo al consultarle si posee conocimiento acerca de los diferentes estilos de aprendizaje del ser humano.

Por lo contrario, el 12,50\% de los encuestados indicó que no están de acuerdo acerca de lo consultado, esto quiere decir que no se encuentran al tanto de los tipos de aprendizaje. 
Es importante conocer acerca de los tipos de aprendizaje, puesto que para las materias técnicas se necesita habilidades particulares, no obstante la población consultada no está formada por gente entendida en la enseñanza, es indispensable conocer su opinión para poder desarrollar el tipo de aprendizaje tecnológico-científico con las herramientas informáticas adecuadas.

\begin{tabular}{lcr}
\multicolumn{1}{c}{ ITEMS } & FRECUENCIA & PORCENTAJE \\
\hline Totalmente de Acuerdo & 58 & $48,33 \%$ \\
De Acuerdo & 50 & $41,67 \%$ \\
No Opina & 9 & $7,50 \%$ \\
En desacuerdo & 3 & $2,50 \%$ \\
Total Desacuerdo & 0 & $0,00 \%$ \\
\multicolumn{1}{r}{ TOTAL } & $\mathbf{1 2 0}$ & $\mathbf{1 0 0 \%}$
\end{tabular}

Cuadro \#21

\section{Item\# 9. Robótica ha ayudado al hombre en todos los campos (educación)}

El 90\% de los informantes está de a favor en la premisa que indica que la Robótica, como herramienta, ha ayudado al hombre en todos los campos de la ciencia, incluido la Educación Superior.

De la misma forma el 2,50\% está en desacuerdo y existe un porcentaje que suma el $7.59 \%$ que No opina al respecto.

En la actualidad donde los procesos se encuentran automatizados y se debe disminuir el riesgo que enfrenta el hombre la robótica ha contribuido positivamente en varias áreas. En el campo de la Educación Superior, además de ser materia, carrera y objeto de estudio, existen robots que ayudan a personas con capacidades especiales y se usan como herramientas didácticas promoviendo la experimentación, investigación y creación de nuevas tecnologías. 


\begin{tabular}{lcr}
\multicolumn{1}{c}{ ITEMS } & FRECUENCIA & PORCENTAJE \\
\hline Totalmente de Acuerdo & 64 & $53,33 \%$ \\
De Acuerdo & 44 & $36,67 \%$ \\
No Opina & 10 & $8,34 \%$ \\
En desacuerdo & 1 & $0,83 \%$ \\
Total Desacuerdo & 1 & $0,83 \%$ \\
\multicolumn{1}{c}{ TOTAL } & $\mathbf{1 2 0}$ & $\mathbf{1 0 0 \%}$
\end{tabular}

\section{Cuadro \#22 \\ Item\# 10. Falta de herramientas didácticas en el aprendizaje de programación}

En el ítem\#10, al igual que el anterior, se tiene una mayoría absoluta que llega al 90\% de la población estudiantil, que opina que hace falta herramientas didácticas y robóticas en al aprendizaje de programación.

Otros datos muestran a informantes que No Opinan del tema en un 8,34\% y estudiantes que están en desacuerdo que suma una minoría de $1,66 \%$

Este apoyo contundente deja claro que las Ciencias de la Educación se encuentran en deuda con el aprendizaje técnico, puesto que no existen técnicas o herramientas específicas para este campo, precisamente en el área de programación. Se evidencia una falta de apoyo didáctico que, en cierto modo, es el objetivo del presente trabajo y problema de investigación.

\begin{tabular}{lcr}
\multicolumn{1}{c}{ ITEMS } & FRECUENCIA & PORCENTAJE \\
\hline Totalmente de Acuerdo & 53 & $44,17 \%$ \\
De Acuerdo & 49 & $40,83 \%$ \\
No Opina & 14 & $11,67 \%$ \\
En desacuerdo & 4 & $3,33 \%$ \\
Total Desacuerdo & 0 & $0,00 \%$ \\
\multicolumn{1}{r}{ TOTAL } & $\mathbf{1 2 0}$ & $\mathbf{1 0 0 \%}$
\end{tabular}

Cuadro \#23

Item\# 11. Armar un robot y manejarlo influye positivamente en el aprendizaje 
Resultado de la aplicación del cuestionario se obtuvo que el $85 \%$ de la población está De Acuerdo en que armar un robot, operarlo y enviar comandos para o programarlo para que ejecute alguna acción influye de manera positiva en el aprendizaje de la materia programación.

El 11,67\% de los informantes No opinan del tema en mención y el resto, es decir, 3,33\% no dieron una respuesta favorable.

El aprendizaje puede producirse de muchas formas y, armar un aparato electrónico, es una de ellas, con esto se desea consolidar la fundamentación teórica de la programación orientada a objetos con una forma práctica y fácilmente perceptible como lo es programar acciones y decisiones.

\begin{tabular}{lcr}
\multicolumn{1}{c}{ ITEMS } & FRECUENCIA & PORCENTAJE \\
\hline Totalmente de Acuerdo & 41 & $34,17 \%$ \\
De Acuerdo & 58 & $48,33 \%$ \\
No Opina & 19 & $15,83 \%$ \\
En desacuerdo & 2 & $1,67 \%$ \\
Total Desacuerdo & 0 & $0,00 \%$ \\
\multicolumn{1}{r}{ TOTAL } & $\mathbf{1 2 0}$ & $\mathbf{1 0 0 \%}$
\end{tabular}

\section{Cuadro \#24 \\ Item\# 12. Resultado estudias indican personas que usan lego desempeñan funciones científicas}

Se consultó a los estudiantes que toman la materia programación orientada a objetos sobre el resultado de un estudio de una Universidad Reconocida que indica que las personas que usan Lego son propensas a realizar labores técnicas y científicas en sus trabajos.

La gran mayoría estuvo de acuerdo, llegando a alcanzar más del $82 \%$ de resultados positivos, divididos en $34,17 \%$ Totalmente de Acuerdo y un 48,33\% que estuvo de Acuerdo. No Opinaron el $15,83 \%$ de la población y 1,67\% estuvo en desacuerdo. 
El estudio revela que lo que se practique y se use como juguete en la edad infantil influye directamente en la profesión. No es desconocido que los bloques para armar o legos son una excelente herramientas que estimula el desarrollo mental de los infantes, por tanto existe la línea Lego Education para los jóvenes y adultos, cuyo objetivo es ayudar en el aprendizaje.

\begin{tabular}{lcr}
\multicolumn{1}{c}{ ITEMS } & FRECUENCIA & PORCENTAJE \\
\hline Totalmente de Acuerdo & 76 & $63,33 \%$ \\
De Acuerdo & 37 & $30,83 \%$ \\
No Opina & 4 & $3,34 \%$ \\
En desacuerdo & 2 & $1,67 \%$ \\
Total Desacuerdo & 1 & $0,83 \%$ \\
\multicolumn{2}{c}{ TOTAL } & $\mathbf{1 2 0}$ \\
\multicolumn{3}{c}{ Cuadro \#25 } \\
Item\# 13. Interesado en ensamblar un robot con su \\
equipamiento y guías
\end{tabular}

El 94,16\% está interesado en armar un robot con su equipamiento y respectivas guías, mientras que el 2,5\% no está de acuerdo y, finalmente, el 3,34\% de los informantes no emite criterio alguno.

En el medio digital que actualmente se está, cada avance tecnológico es importante y la comunidad está abierta a la aplicación de la informática en su vida cotidiana.

El apoyo extremamente mayoritario que se tiene para experimentar con robótica, sensores, motores, programas, etc. es la respuesta a una sociedad llena de información digital con sed de conocimiento y dispuesta a innovar, en donde la tecnología forma parte de la vida de las personas y están enraizadas en solventar o ayudar el día a día de la gente, convirtiéndose en imprescindible en un día normal. 


\begin{tabular}{|c|c|c|}
\hline ITEMS & FRECUENCIA & PORCENTAJE \\
\hline Definitivamente $\mathrm{Si}$ & 84 & $70,00 \%$ \\
\hline $\mathrm{Si}$ & 33 & $27,50 \%$ \\
\hline No Opina & 3 & $2,50 \%$ \\
\hline No & 0 & $0,00 \%$ \\
\hline Definitivamente No & 0 & $0,00 \%$ \\
\hline TOTAL & 120 & $100 \%$ \\
\hline
\end{tabular}

EL 97.5\% de los informantes desean conocer como ensamblar un robot de Lego con instrucciones gráficas en tercera dimensión, el $2.5 \%$ decidió no opinar nada sobre el tema en cuestión y ningún estudiante estuvo en desacuerdo.

Lego lidera el mercado de los bloques armables y ha creado el Mindstorms como respuesta a los requerimientos de robot caseros y de fácil uso.

Las instrucciones que se encuentren en internet o en los folletos físicos indican como armar cada aparato. Una de las últimas innovaciones del fabricante es una consola en donde se puede crear, moldear y diseñar un robot, con las piezas proporcionadas (por el mismo fabricante) en tercera dimensión, con esto facilita el ensamblaje y brinda un mundo infinito de imaginación.

\begin{tabular}{lcr} 
& FRECUENCIA & PORCENTAJE \\
\hline Definitivamente Si & 67 & $55,84 \%$ \\
Si & 48 & $40,00 \%$ \\
No Opina & 3 & $2,50 \%$ \\
No & 1 & $0,83 \%$ \\
Definitivamente No & 1 & $0,83 \%$ \\
\multicolumn{1}{r}{ TOTAL } & $\mathbf{1 2 0}$ & $\mathbf{1 0 0 \%}$
\end{tabular}

Cuadro \#27

Item\# 15. Información acerca de lego Mindstorms y su aplicación en la educación 
En la pregunta en donde se consulta si desean obtener más información acerca de Lego Mindstorms y su aplicación en las ciencias de la educación se cuenta con una mayoría del 95,84\% a favor en donde el 55,84\% indica que definitivamente si desearía y el $40 \%$ responde de manera afirmativa.

Como se ha podido ver, el uso de nuevas herramientas didácticas y tecnológicas que apoyen el proceso de enseñanza-aprendizaje es bien recibido por parte de los estudiantes, puesto que les permitirá desempeñarse mejor el aula de clase e incentivará la investigación, desarrollo e innovación.

Es importante recalcar que Lego Mindstorms combina robótica con programación de acciones, esto les ayuda a los estudiantes a aplicar y esclarecer la teoría de forma visual y entretenida.

\begin{tabular}{l|c|r}
\multicolumn{1}{r}{ ITEMS } & FRECUENCIA & PORCENTAJE \\
\hline Definitivamente Si & 3 & $2,50 \%$ \\
\hline $\mathrm{Si}$ & 8 & $6,67 \%$ \\
No Opina & 11 & $9,17 \%$ \\
\hline No & 85 & $70,83 \%$ \\
\hline Definitivamente No & 13 & $10,83 \%$ \\
\multicolumn{1}{r|}{ TOTAL } & $\mathbf{1 2 0}$ & $\mathbf{1 0 0 \%}$
\end{tabular}

\section{Cuadro \#28}

Item\# 16. conocimiento de lenguajes de programación que soporta lego Mindstorms

La opinión de los informantes fue mayoritaria al desconocimiento en este ítem, en cuanto sólo el 9,17\% si conocía sobre los lenguajes de programación que se usa en Lego Mindstorms, mientras que el 81,66\% respondió lo contrario. 
Un $9,17 \%$ de los informantes prefirieron No opinar por lo que se tiene más del $90 \%$ el cual aportó sus consideraciones a la encuesta.

Lego Mindstorms soporta diversos lenguajes de programación como C, C\#, Java, entre otros lo cual es importantísimo, ya que el hecho de no estar atado a una sola plataforma crea diversidad de conocimiento y opciones al momento de diseñar, con esto no es relevante el lenguaje a usar, sino como se puede aplicar la herramienta al proceso de enseñanza-aprendizaje de la materia Orientada a Objetos.

\begin{tabular}{|c|c|c|}
\hline ITEMS & FRECUENCIA & PORCENTAJE \\
\hline Totalmente de Acuerdo & 35 & $29,17 \%$ \\
\hline De Acuerdo & 45 & $37,50 \%$ \\
\hline No Opina & 39 & $32,50 \%$ \\
\hline En desacuerdo & 1 & $0,83 \%$ \\
\hline Total Desacuerdo & 0 & $0,00 \%$ \\
\hline TOTAL & 120 & $100 \%$ \\
\hline
\end{tabular}

Se consultó con los estudiantes si piensan que el aprendizaje se facilita con el uso de Lego Mindstorms con su respectiva guía y se obtuvo el 66,67\% de apoyo, diferenciado en 29,17\% que se encontraban Totalmente de Acuerdo y el resto, o sea 37,50\%, está de Acuerdo.

Además de los resultados favorables se obtuvo que un 32.50 No opinó y el 0,83\% estuvo en desacuerdo.

Con la encuestas a los estudiantes se puede confirmar, al igual que resultó con los docentes, el apoyo mayoritario a la utilización de Lego Mindstorms como herramienta robótica didáctica para el aprendizaje de Programación Orientada a Objetos en la Universidad de Guayaquil. 
Juan C. Ramos-Romero; Janet E. Pazmiño-Ramírez; Silvia A. Medina-Anchundia

\begin{tabular}{lcr}
\multicolumn{1}{c}{ ITEMS } & FRECUENCIA & PORCENTAJE \\
\hline Totalmente de Acuerdo & 55 & $45,83 \%$ \\
De Acuerdo & 50 & $41,67 \%$ \\
No Opina & 14 & $11,67 \%$ \\
En desacuerdo & 1 & $0,83 \%$ \\
Total Desacuerdo & 0 & $0,00 \%$ \\
\multicolumn{1}{r}{ TOTAL } & $\mathbf{1 2 0}$ & $\mathbf{1 0 0 \%}$
\end{tabular}

Cuadro \#30

Item\# 18. Apoyo al uso de lego Mindstorms para aprender programación orientada a objetos

En la última pregunta de la encuesta a los estudiantes se puede evidenciar que el 87,50\% de los encuestados apoyan el uso de Lego Mindstorms en el aprendizaje de Programación Orientada a Objetos.

El 11,67\% de los informantes se abstuvo de opinar del tema y, finalmente, el 0,83\% se encontró en desacuerdo.

El uso de Lego Mindstorms en el proceso metodológico de clase es muy recomendable en el proceso de enseñanza-aprendizaje, así lo piensan la mayoría de los estudiantes encuestados, por lo tanto aprueban la hipótesis planteada dentro del trabajo de investigación.

\section{Conclusiones.}

Una vez realizado el estudio estadístico y mediante el respectivo análisis de los gráficos se ha llegado a la conclusión que el 50\% de los estudiantes piensan que los conceptos de programación orientada a objetos por el hecho de ser abstractos son complejos de aprender, en el ítem \#1, por lo tanto, se concluye la existencia de este problema educativo. 
Además, el $50 \%$ de los estudiantes afirma tener conocimiento teórico de los pilares fundamentales de la programación orientada a objetos, el 18\% prefiere no opinar en el ítem \#2, por lo tanto, pese a tener conocimiento indican que sus conceptos con complejos.

El lenguaje orientado a objetos más relevante es el lenguaje Open Source denominado Java por el creador Sun Microsystem, eso determinan el $65 \%$ los estudiantes y el $80 \%$ de los docentes consultados mediante el cuestionario.

Los estudiantes en el ítem \# 6 piensan que determinar los fundamentos de Programación Orientada a Objetos facilita los procesos de enseñanza - aprendizaje de la materia, según el resultado porcentualmente el $86,67 \%$ está de acuerdo, por lo que se puede concluir lo expuesto anteriormente.

La falta de guías especializadas por materias influye en el aprendizaje y rendimiento de los estudiantes, eso afirma casi el 90\% de los estudiantes en el ítem \#7.

Este alto porcentaje alerta que hacen falta guías especializadas en una materia, como documentación técnica o manuales conocidos en inglés como "How to do" o "Cómo se hace".

El $90 \%$ de los estudiantes y el $80 \%$ de los docentes afirman que la robótica, en sus distintos aspectos, ha ayudado al hombre incluido el campo de la educación.

Este resultado ayuda a concluir que se tiene en la robótica un aliado estratégico para cubrir ciertas áreas en donde el hombre aún necesite ayuda, y la Educación Superior es una de esas, y en el presente trabajo se lo está enfocando a la Programación Orientada a Objetos como una materia de tecnología que complementaría cualquier aplicación robótica que se implemente. 
Juan C. Ramos-Romero; Janet E. Pazmiño-Ramírez; Silvia A. Medina-Anchundia

Se concluye y afirma un mayoritario $67 \%$ de estudiantes y el $100 \%$ de los docentes que la Programación Orientada a Objetos sería más fácil de asimilar mediante una herramienta como Lego Mindstorms y su respectiva guía de aprendizaje. Esto apoya significativamente a la propuesta de realizar una guía de aprendizaje de Programación Orientada a Objetos usando la herramienta Lego Mindstorms, puesto que aparte de facilitar la generación de conocimiento, incentiva y motiva a los estudiantes a la investigación y exploración de nuevas áreas y la aplicación del mismo.

Se cuenta con un apoyo total de los docentes y con el $87.5 \%$ de los estudiantes en el uso de Lego Mindstorms para aprender Programación Orientada a Objetos, así lo indica el último ítem en cada uno de los respectivos cuestionarios.

La respuesta afirmativa y el evidente apoyo mayoritario y total en el caso de los docentes da la razón y justifica la propuesta de elaborar una guía para la aplicación de la herramientas antes mencionada en el proceso de enseñanza-aprendizaje de Programación Orientada a Objetos, por lo que beneficiará a la comunidad universitaria innovando y elevando el rendimiento de los estudiantes, esto aportará significativamente a la excelencia que es el objetivo al cual se necesita llegar.

\section{Agradecimiento}

Doy gracias a Dios, en primer lugar, luego a mis padres por su apoyo incondicional, a mi Tutor en el que siempre encontré una guía y a todas las personas que directa o indirectamente contribuyeron en que avance y culmine el presente trabajo, especialmente a esa personita especial que me apoyó durante todo el desarrollo del mismo... 


\section{Bibliografía.}

Arnedo, José Moreno (2007). Programación Orientada a Objetos. México, México. S.L. Editorial UOC.

Bagnall, Brian (2014). Maximum LEGO EV3: Bringing Robots to Life with Java (LEGO Mindstorms EV3). EE.UU.: Variant Press.

Benedettelli, Daniele (2013). The LEGO MINDSTORMS EV3 Laboratory: Build, Program, and Experiment with Five Wicked Cool Robots! San Francisco, EE.UU.: [s.n].

Budd, Timoty (2002). Introducción a la Programación Orientada a Objetos. Argentina. Addison-Wesley.

Campos, Yolanda (2009). ¿Cómo hacer el aprendizaje significativo? Quito, Ecuador: Grupo Santillana.

Craig, John J. (2006). Robótica (3era Edición) México: Pearson Education, Prentice Hall.

Gonzalez, José Francisco (1995). Al día en una hora ROBOTICA. México: Anaya Multimedia.

Graham, Ian (2000) Métodos Orientados a Objetos. Madrid: Ediciones Días de Santos.

Karch, Marziah(2014). Build and Program Your Own LEGO Mindstorms EV3 Robots (1era Edición). EE.UU.: Que Publishing.

Kee, Damien (2013). Classroom Activities for the Busy Teacher: EV3. EE.UU.: CreateSpace Independent Publishing Platform.

Kmiec, Pawel (2012). The Unofficial LEGO Technic Builder's Guide. China: No Starch Press.

López, Leobrado (2007) Metodología de la Programación Orientada a Objetos. México. Alfaomega

Mark Rollins (2014). Beginning LEGO MINDSTORMS EV3: (B\&W). EE.UU.: Apress.

Park, Eun Jung (2014). Exploring LEGO Mindstorms EV3: Tools and Techniques for Building and Programming Robots. EE.UU.: Wiley

Pimienta, Julio Prieto (2012). Estrategias de Enseñanza-aprendizaje. México: Pearson Education.

Reyes, Fernando (2005). Robótica, Control de Robots Manipuladores. México: Alfaomega.

Rollins, Mark (2013). LEGO Technic Robotics (Technology in Action). New York, EE.UU. Apress.

Romero, Costas Matías (2012). Robótica: entra al mundo de la inteligencia artificial (1era edición). Buenos Aires, Argentina: Educar S.E.

Sabino, Carlos (2011). El Proceso de Investigación. Caracas, Venezuela: Editorial Panapo.

Wang, Paul S. (2000). Java con Programación Orientada a Objetos y Aplicaciones en la www. México: International Thomson Editores. 